\title{
Self-Organized Patterning of an Insulator-on-Metal System by Surface Faceting and Selective Growth: $\mathrm{NaCl} / \mathrm{Cu}(211)$
}

\author{
S. Fölsch, A. Helms, S. Zöphel, J. Repp, G. Meyer, and K. H. Rieder \\ Institut für Experimentalphysik, Freie Universität Berlin, Arnimallee 14, D-14195 Berlin, Germany
}

(Received 11 August 1999)

\begin{abstract}
We report experimental results on an insulator-on-metal system which is inherently unstable against lateral pattern formation on the nanometer scale. $\mathrm{NaCl}$ deposition on $\mathrm{Cu}(211)$ at substrate temperatures $>300 \mathrm{~K}$ leads to faceting into (311) and (111) facets and selective $\mathrm{NaCl}$ growth on (311) facets only, thereby creating alternating stripes of bare $\mathrm{Cu}$ and $\mathrm{NaCl}$-covered areas. The mesoscopic restructuring process is brought about by (1) the tendency to form (100)-terminated $\mathrm{NaCl}$ layers, (2) epitaxial matching between $\mathrm{NaCl}(100)$ and $\mathrm{Cu}(311)$, and (3) sufficient mobility of the $\mathrm{Cu}$ substrate surface.
\end{abstract}

PACS numbers: 68.35.Bs, 61.14.Hg, 61.16.Ch, 68.55.-a

Besides its general importance to device miniaturization, the fabrication of nanometer-scale surface structures enables one to alter physical properties of matter by confining electrons to dimensions comparable to their wavelength. Atomic manipulation by scanning tunneling microscopy (STM) [1,2] offers the unique possibility to built up prototype structures atom by atom. To create large-scale arrays of nanostructures, growth techniques have to be employed which take advantage of selforganized processes induced by anisotropic surface diffusion [3], strain relaxation [4], or selective growth due to reconstructions [5], dislocations [6], steps [7] or facets [8] at a substrate surface. This concept has been widely used to grow quantum dots and quantum wires of semiconductors and metals. In contrast, nothing is known so far on how lateral structuring of wide-band gap insulators may be achieved by growth-mediated processes. This material class is still a missing link in the attempt to cover the whole range of electronic properties for the fabrication of nm-scale surface structures. In the following, we present for the first time an insulator-on-metal growth system which meets the requirement of self-organized lateral pattern formation: When $\mathrm{NaCl}$ is deposited on a $\mathrm{Cu}(211)$ host, massive surface faceting occurs resulting in a periodic $1 \mathrm{D}$ hill-and-valley structure with $\mathrm{NaCl}$ growing selectively on only one of the two facet orientations present. A modulated surface evolves which consists of alternating nanostripes of bare $\mathrm{Cu}$ and $\mathrm{NaCl}$-covered areas. Growth temperature control allows one to vary the stripe spacing over 1 order of magnitude from $30 \AA$ to $230 \AA$. In this way, a surface pattern is formed which is periodic on the length scale relevant for electron confinement. It is shown that the preference to form (100)terminated layers and the attempt to optimize interfacial lattice matching between the $\mathrm{NaCl}$ layer and the $\mathrm{Cu}$ template is the energetic driving force for the mesoscopic restructuring process observed here.

The present results are based on structural investigations with high-resolution LEED (SPALEED) and STM. A Cu(211) crystal was cleaned in UHV by alternate $\mathrm{Ne}^{+}$ sputtering and annealing at $750 \mathrm{~K}$. After repeated prepa- ration cycles a sharp $(1 \times 1)$ LEED pattern indicated a well-ordered $\mathrm{Cu}(211)$ surface with a defect-free length of $\sim 500 \AA$ as deduced from the measured half-width of the specular LEED beam. At this stage no residual contamination was detectable by $\mathrm{x}$-ray photoelectron spectroscopy (XPS). $\mathrm{NaCl}(99.95 \%$ purity) was evaporated from a $\mathrm{Al}_{2} \mathrm{O}_{3}$ crucible at 2 monolayer/min (ML/min) [9] and growth temperatures of 300-600 K. It was confirmed by XPS that stoichiometry of the $\mathrm{NaCl}$ deposit is preserved over the entire temperature and coverage range investigated.

$\mathrm{Cu}(211)$ is a vicinal surface with (111) terraces separated by intrinsic (100) single steps along the [011] direction. The resulting surface geometry is shown in the lower part of the top view scheme in Fig. 1 (nonequivalent substrate atoms are numbered): The surface symmetry is described by a primitive rectangular unit cell and the intrinsic step separation measures $6.25 \AA$. In STM images these intrinsic steps show up as a clear surface corrugation [2]. This starting surface drastically restructures upon $\mathrm{NaCl}$ submonolayer deposition if the growth temperature exceeds $\sim 300 \mathrm{~K}$. The temperature onset of the restructuring process is in the range in which our STM observations of bare $\mathrm{Cu}(211)$ indicate emerging kink site mobility. In detail, the initially flat substrate is transformed into a ridgelike surface topography whose ridges are aligned with the (100) step direction. The resulting facet structure can be analyzed by LEED if one maps a vertical cut of reciprocal space with contains the scattering vector component $K_{\perp}$ normal to the macroscopic surface and the parallel component $K_{\|}$along [11 $\left.\overline{1}\right]$ (i.e., perpendicular to the intrinsic steps). Figure 2 shows a gray scale graph of the LEED intensity $I\left(K_{\perp}, K_{\|}\right)$for $0.6 \mathrm{ML} \mathrm{NaCl}$ deposited at $490 \mathrm{~K}$. The (10), (00), and (10) lattice rod positions of the $\mathrm{Cu}(211)$ substrate are marked by vertical lines together with the respective (533), (422), and (733) Bragg points. The substrate lattice rod spacing corresponds to the reciprocal spacing of intrinsic steps and equals $1.005 \AA^{-1}$ (note that the $K_{\perp}$ axis is scaled by a factor of 0.5 with respect to the $K_{\|}$axis). As evident, the diffracted intensity is confined to two separate sets of 


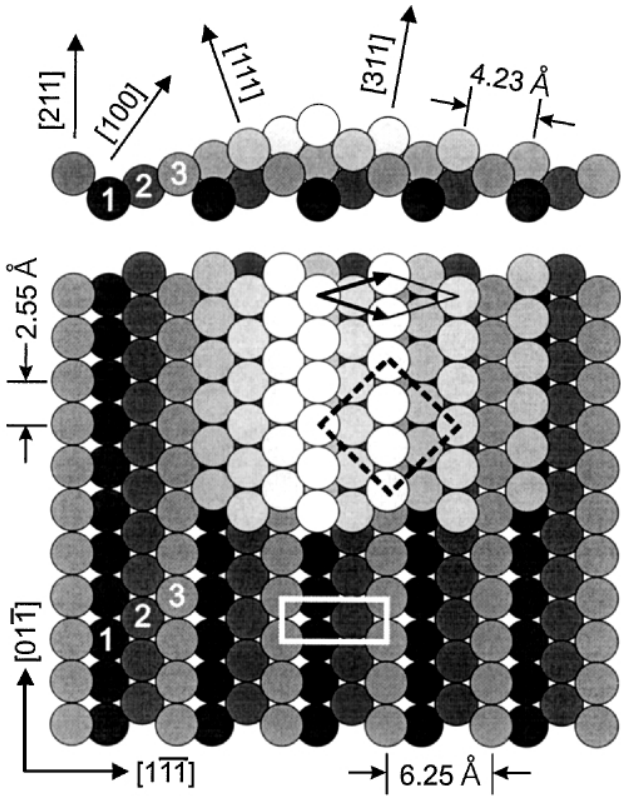

FIG. 1. Sphere model of the $\mathrm{Cu}(211)$ substrate with rectangular unit cell (lower part of top view scheme) and a ridge built up by a (111)/(311) facet pair (side view and upper part of top view scheme). The rhombic unit cell of the $\mathrm{Cu}(311)$ plane (cf. respective unit vectors and full black line) and the cubic unit cell of the $\mathrm{NaCl}(100)$ layer containing two molecules (dashed line) are also shown; nonequivalent $\mathrm{Cu}(211)$ surface atoms are numbered.

inclined facet rods which intersect at the Bragg points and run along the (311) and (111) direction, respectively. In contrast, the intensity is extinguished along the substrate rod positions indicating completion of the faceting process at this coverage. The (311) rods are straight and remain sharp irrespective of the scattering condition, indicating a high degree of long-range order and flatness of the (311) facets. The (111) rod in Fig. 2, on the other hand, is split up into discrete segments tilted towards the macroscopic surface normal direction. This indicates vicinal misorientation [10] of the (111) facets: The observed 5.7 tilt yields a reduced mean facet inclination of $13.8^{\circ}$ [cf. $19.5^{\circ}$ inclination for flat (111) facets] at this stage of coverage and growth temperature, which is equivalent to (11 9 9) vicinal orientation. The present ridge structure thus corresponds to an alternation of flat (311) facets and vicinal (111) facets. Figure 1 visualizes how these facet orientations are related to the $\mathrm{Cu}(211)$ surface [for simplicity a flat (111) facet is shown]: The facets are built up by incorporating $\mathrm{Cu}$ atom rows along the intrinsic step direction. For the resulting (311) facet this leads to a rhombic unit cell (cf. respective unit vectors and full black line) and a reduced row separation of $4.23 \AA$. It is evident from Fig. 1 that the facet formation process requires considerable $\mathrm{Cu}$ mass transport. Hence, one expects a sensitive dependence of the ridge size on substrate temperature (i.e., on adatom mobility) during deposition. It was shown in Ref. [11] that coherent scattering at a periodic facet array yields a finite-size splitting $\Delta K_{\|}$

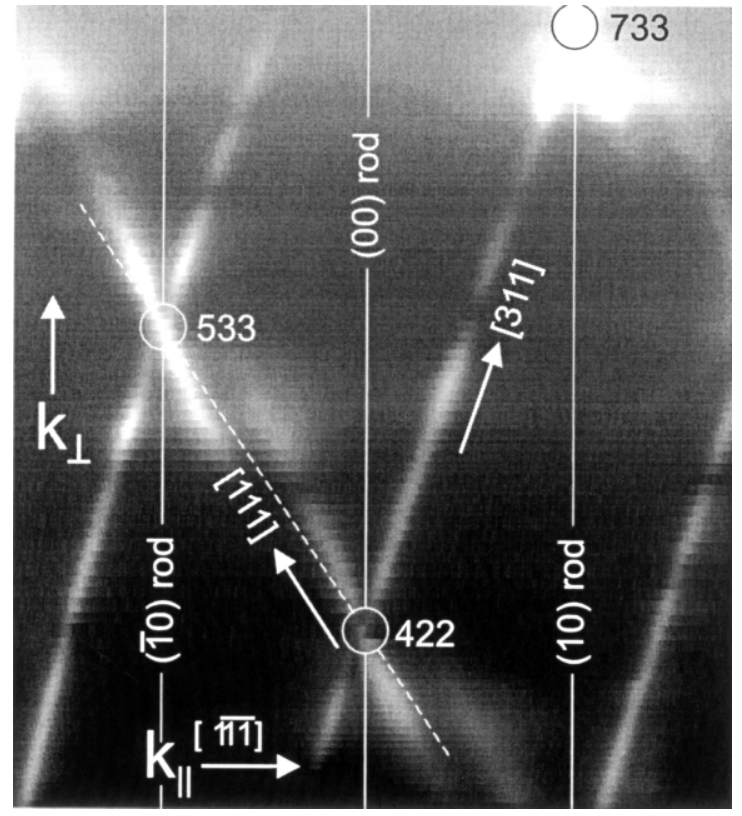

FIG. 2. Gray scale graph of the LEED intensity $I\left(K_{\perp}, K_{\|}\right)$for $0.6 \mathrm{ML} \mathrm{NaCl}$ deposited at $490 \mathrm{~K}$ with $K_{\|}$pointing along [1 $\left.\overline{1} \overline{1}\right]$ (perpendicular to intrinsic steps). The spacing of the $\mathrm{Cu}(211)$ substrate lattice rods (vertical lines) measures $1.005 \AA^{-1}$; related Bragg points are denoted by circles. The diffracted intensity is confined to two separate sets of inclined facet rods indicating the presence of flat (311) facets and vicinal (111) facets. The $K_{\perp}$ axis is scaled by 0.5 with respect to the $K_{\|}$axis.

of the specular LEED beam close to the Bragg condition, whose corresponding length $2 \pi / \Delta K_{\|}$in real space is associated with the mean separation of equivalent facets $\langle\Lambda\rangle$. The spatial periodicity of the facet array thus gives rise to a momentum transfer $\Delta K_{\|}$ which can be extracted from the LEED beam profile. For the present facet structure, we indeed observe sharp and pronounced satellites of the specular beam close to the Bragg condition, revealing a continuous increase of $\langle\Lambda\rangle$ with growth temperature ranging from $\sim 30 \AA$ at $300 \mathrm{~K}$ to $\sim 230 \AA$ at $600 \mathrm{~K}$. It is thus possible to adjust the mean lateral ridge distance by growth temperature.

The driving force of the restructuring process is manifested in the crystallographic relationship between the $\mathrm{NaCl}$ layer and the $\mathrm{Cu}$ template: $\mathrm{NaCl}$ grows selectively on the (311) facets and forms an epitaxial singledomain layer with (100) termination [12]. Figure 3 shows a LEED pattern for 0.6 ML coverage taken along the [311] direction, i.e., the (00) spot in the center of the pattern corresponds to the specular beam of the (311) facets. Apart from the integral-order spots of the $\mathrm{Cu}(311)$ plane (cf. rhombic unit cell, full line) a coincident superstructure with a distorted cubic unit cell (dashed line) is observed. The labeling of superstructure spots (broken indices in Fig. 3$)$ refers to the $(1 \times 1)$ symmetry of $\mathrm{Cu}(311)$. In real space the observed superstructure corresponds to a distorted simple cubic $\mathrm{NaCl}$ cell; see dashed 


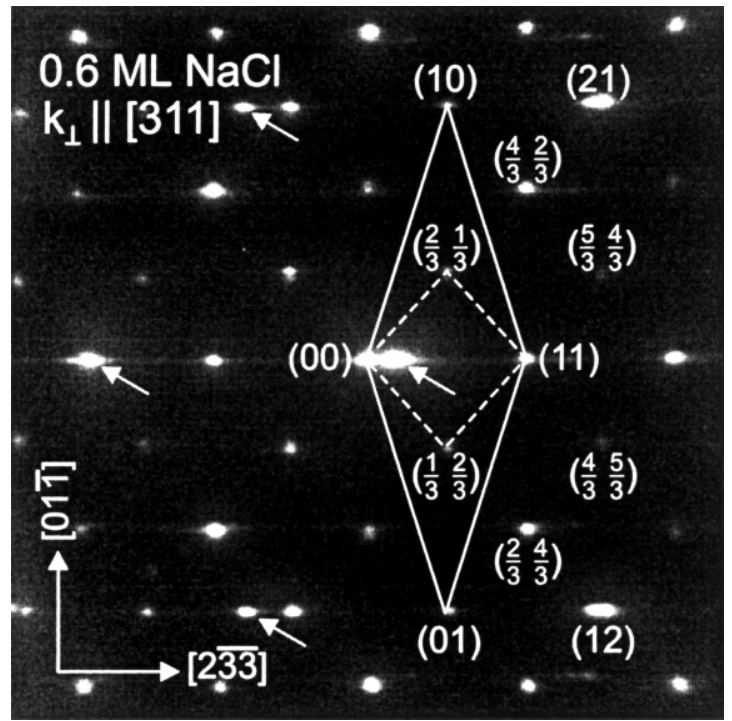

FIG. 3. LEED pattern taken at $120 \mathrm{eV}$ and $0.6 \mathrm{ML}$ coverage, the (00) spot in the center corresponds to the specular beam of the (311) facets. Apart from integral-order spots of the $\mathrm{Cu}(311)$ plane (rhombic unit cell, full line) a coincident superstructure is observed (distorted cubic unit cell, dashed line); superstructure spots are indexed with respect to the $(1 \times 1)$ symmetry of $\mathrm{Cu}(311)$. Diffraction spots marked by arrows originate from the vicinal (111) facets.

line in Fig. 1: The cell diagonals match with the twofold row separation of the $\mathrm{Cu}(311)$ plane $(8.46 \AA)$ and the threefold interatomic distance within the rows $(7.66 \AA)$, their lengths are thus comparable with the $\mathrm{NaCl}$ bulk value of $\sqrt{2} a_{0}=7.98 \AA$. Hence, $\mathrm{NaCl}$ forms a (100)terminated overlayer with its $\langle 011\rangle$ azimuthal directions parallel and perpendicular to the $\mathrm{Cu}$ atom rows.

Complementary STM data prove the selectivity of the growth process and the spatial anisotropy of the evolving surface structure. Figures 4(a) and 4(b) illustrate the initial stage after depositing $0.1 \mathrm{ML}$ at $370 \mathrm{~K}$. The detailed section [4(a) $120 \AA \times 80 \AA$ ] shows the intrinsic steps of a plain $\mathrm{Cu}(211)$ region still present at this coverage (left), the ascending (311) facet with epitaxial $\mathrm{NaCl}(100)$ overlayer (center), and the corrugation of the descending vicinal $\mathrm{Cu}(111)$ facet (right) which is (533) oriented at this stage. By comparing the surface area occupied by (311) facets with the amount of deposited material we confirmed that the (311) facets are overgrown by a single $\mathrm{NaCl}$ monolayer. The enlarged scale image [4(b) $400 \AA \times 400 \AA$ ] shows two discrete ridges preferably growing along the direction of intrinsic (100) steps. Their (311) facets are entirely flat and uniform in width. The perfect 2D growth inferred from the flatness of the $\mathrm{NaCl}$-covered facets indicates that the present epitaxial orientation is energetically highly favorable, i.e., the interfacial energy between the $\mathrm{NaCl}(100)$ overlayer and the $\mathrm{Cu}$ template is minimized for (311) substrate orientation. Obviously, this energetic preference is the driving force of the restructuring process observed here. It competes with two diffusion limited processes, namely, the surface
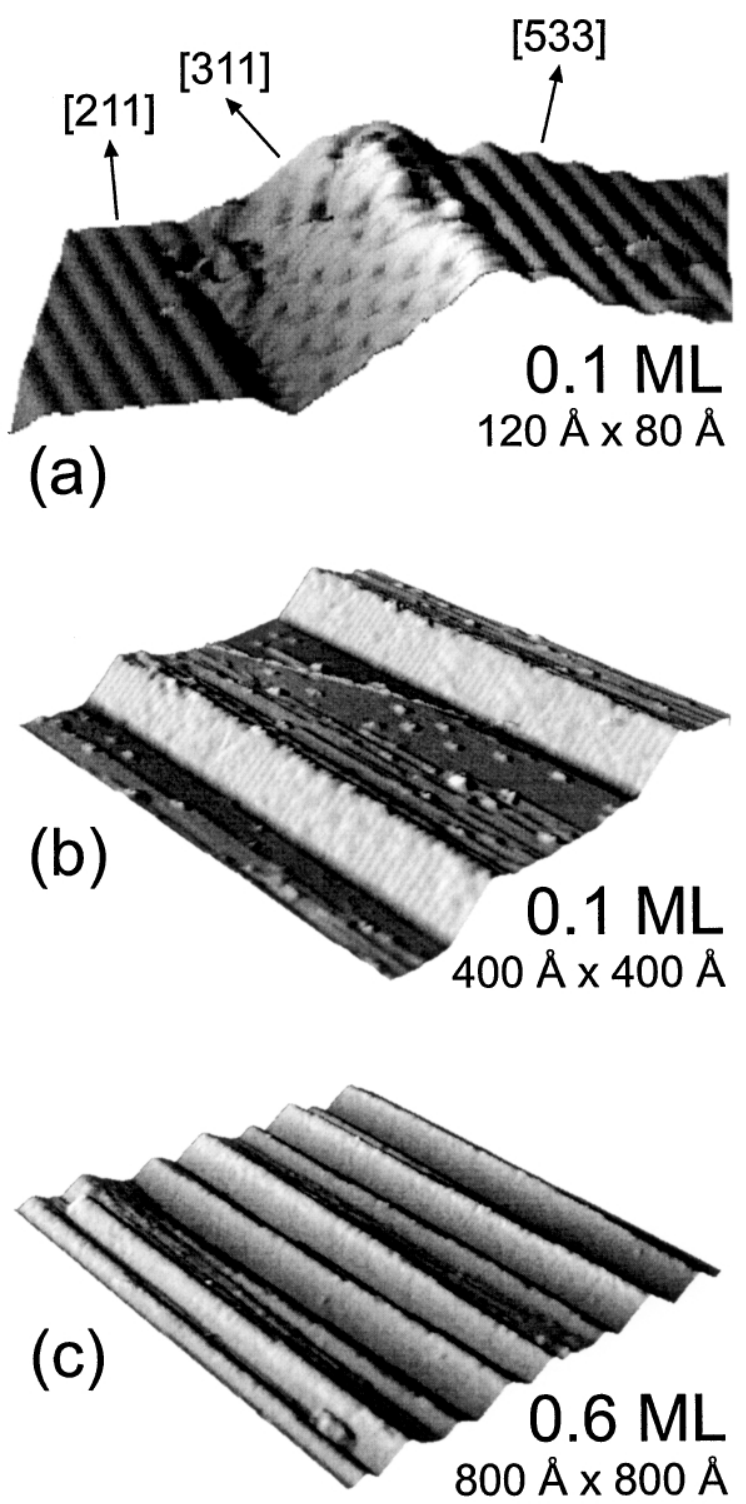

FIG. 4. (a) STM image $(120 \AA \times 80 \AA)$ at $0.1 \mathrm{ML}$ coverage demonstrating selective $\mathrm{NaCl}$ growth on the (311) facet (center); the corrugation (left and right) shows a $\mathrm{Cu}(211)$ surface region and the intrinsic $\mathrm{Cu}$ steps of a vicinal (111) facet which is (533) oriented at this stage. (b) Enlarged area scan $(400 \AA \times 400 \AA)$ at 0.1 ML coverage showing two $\mathrm{NaCl}$-covered (311) facets uniform in width and preferably growing along the intrinsic step direction. (c) STM image $(800 \AA \times 800 \AA)$ after completion of the faceting process at $0.6 \mathrm{ML}$ coverage showing the spatial anisotropy and the regularity of the resulting surface topography. All images relate to $370 \mathrm{~K}$ growth temperature and tunneling parameters of $\sim 100 \mathrm{pA}$ and $-1 \mathrm{~V}$ sample bias.

migration of $\mathrm{NaCl}$ and the mobility of the substrate surface itself. We now address potential mechanisms which cause the anisotropic growth behavior deduced from the elongated facet shape in Fig. 4(b). First of all, the $\mathrm{Cu}$ adatom mobility is expected to be anisotropic in character owing to the uniaxial surface symmetry. Since selfdiffusion studies for $\mathrm{Cu}(211)$ are not available to date, we refer to effective medium theory calculations of sur- 
face defects on $\mathrm{Cu}(111)$ [13] to derive a rough estimate: According to Ref. [13] the activation energy for adatom diffusion along and across $\langle 01 \overline{1}\rangle$ steps amounts to 0.228 and $0.365 \mathrm{eV}$, respectively. In the relevant temperature range of $300-600 \mathrm{~K}$ the hopping rate along steps is thus $10^{2}-10^{1}$ times larger compared to the hopping rate across steps (assuming comparable prefactors [14]). This leads to mass transport preferably along the step edge direction. A further argument for anisotropic growth follows from the balance between the energetic constraint to form (311) facets and the kinetic limitation of $\mathrm{Cu}$ mass transport required. To show this, one has to consider the total volume of a ridge built up by an ascending (311) facet and a descending (111) facet (cf. Fig. 1). The (311) facet length along the atom row direction of the $\mathrm{Cu}(311)$ plane enters linearly into the ridge volume. The facet width perpendicular to the rows, on the other hand, yields a quadratic contribution since it increases both the total width and the height of the ridge. Elongation along the row direction thus minimizes the material transport required to create (311) facets, inducing the ridges to grow preferably along the direction of intrinsic (100) steps. With increasing coverage the unidirectional ridge growth proceeds until the whole surface is transformed into a coherent array of (311) and vicinal (111) facets. We observe completion of the faceting process after depositing $\sim 0.6 \mathrm{ML} \mathrm{NaCl}$. This critical coverage is consistent with the relative surface area occupied by (311) facets amounting to a value of 0.58 if one takes into account the $13.8^{\circ}$ inclination of the vicinal (111) facets at this final stage. The remaining misorientation of the (111) facets corroborates the fact that the final surface topography is not a pure equilibrium structure, instead, it is reached via a diffusion limited approach towards equilibrium. Figure 4(c) shows the resulting surface structure after depositing $0.6 \mathrm{ML}$ at $370 \mathrm{~K}$. The mean lateral ridge distance extracted from the STM data is $\langle\Lambda\rangle=110 \AA$ at this growth temperature (in agreement with the LEED analysis). Large area STM scans reveal a mean (311) facet length along the intrinsic step direction in the range of typically $1 \mu \mathrm{m}$. Hence, one finally ends up with a surface which is modulated with respect to both topography and chemical composition.

In summarizing, we emphasize that the self-organized pattern formation observed here is remarkable for several reasons. First, it is connected with dramatic surface restructuring induced by the $\mathrm{NaCl}$ deposit. This is in sharp contrast to the general growth behavior of alkali halides on close-packed metal surfaces known to date: Growth studies on $\mathrm{Al}(100), \mathrm{Al}(111)$ [15], and $\mathrm{Cu}(111)$ [16] suggest an inert overlayer that moderately interacts with the metal substrate. In the present case we find an exorbitantly strong energetic preference for epitaxial matching between (100)-terminated $\mathrm{NaCl}$ and $\mathrm{Cu}(311)$. Consistently, supplementary experiments show that perfectly flat $\mathrm{NaCl}$ layers with defect-free domain sizes in the $\mu \mathrm{m}$ range can be grown on a $\mathrm{Cu}(311)$ crystal. The central finding of the present work, however, is that this energetic preference plays the key role in the observed restructuring process of the $\mathrm{Cu}(211)$ surface: It drives the formation of (311) facets which are selectively overgrown by (100)-terminated $\mathrm{NaCl}$ and thus creates a regularly patterned surface. Such a prestructured template with alternating insulator stripes and bare metal areas is a prerequisite substrate for lateral structuring of subsequently deposited material by selective decoration. This concept has been proposed previously by other authors [17] as a potential method to fabricate, e.g., magnetic nanostructures. It is suggested that the basic ingredients of the patterning process, namely, the interplay between substrate mobility and energetic constraints due to interfacial matching, are of general applicability also to the surface structuring of other insulator-on-metal combinations.

This research was supported by the VolkswagenStiftung (I/72 417) and the Deutsche Forschungsgemeinschaft (RI 472/3-2, Sfb 290/TPA5).

[1] D. M. Eigler and E. K. Schweizer, Nature (London) 344, 524 (1990).

[2] G. Meyer, S. Zöphel, and K. H. Rieder, Phys. Rev. Lett. 77, 2113 (1996).

[3] H. Röder et al., Nature (London) 366, 141 (1993).

[4] J. Tersoff and R. M. Tromp, Phys. Rev. Lett. 70, 2782 (1993).

[5] D. D. Chambliss, R. J. Wilson, and S. Chiang, Phys. Rev. Lett. 66, 1721 (1991).

[6] H. Brune, Surf. Sci. Rep. 31, 121 (1998).

[7] P. M. Petroff, A.C. Gossard, and W. Wiegmann, Appl. Phys. Lett. 45, 620 (1984); M. Mundschau, E. Bauer, and W. Swiech, J. Appl. Phys. 65, 581 (1989); Y. W. Mo and F. J. Himpsel, Phys. Rev. B 50, 7868 (1994).

[8] R. Nötzel et al., Phys. Rev. Lett. 67, 3812 (1991); C. Teichert et al., Appl. Phys. Lett. 74, 588 (1999).

[9] $1 \mathrm{ML}$ refers to the density per area of $\mathrm{NaCl}(100)[6.3 \times$ $\left.10^{18} \mathrm{~m}^{-2}\right]$.

[10] M. Henzler, Appl. Surf. Sci. 11/12, 450 (1982).

[11] J. Wollschläger, E. Z. Luo, and M. Henzler, Phys. Rev. B 57, 15514 (1998).

[12] For bulk $\mathrm{NaCl}$ the (100) plane is the only stable equilibrium crystal face due to pronounced minimization of the surface free energy [see G.C. Benson, J. Chem. Phys. 35, 2113 (1961)].

[13] P. Stoltze, J. Phys. Condens. Matter 6, 9495 (1994).

[14] T. T. Tsong, J. Liu, and C.W. Wu, in Physics and Chemistry of Finite Systems: From Clusters to Crystals, edited by P. Jena (Kluwer, Dordrecht, The Netherlands, 1992).

[15] W. Hebenstreit et al., Surf. Sci. 424, L321 (1999).

[16] R. Bennewitz et al., Surf. Sci. 438, 289 (1999).

[17] F. J. Himpsel et al., Superlattices Microstruct. 15, 237 (1994). 